\title{
ESTUDIO DE ALGUNAS VARIABLES QUE AFECTAN LA COMPRENSIÓN DE TEXTOS DE FÍSICA
}

\author{
MACÍAS, ASCENSIÓN, CASTRO, JOSÉ I. y MATURANO, CARLA I. \\ Instituto de Investigaciones en Educación en las Ciencias Experimentales (IIECE). \\ Facultad de Filosofía, Humanidades y Artes. Universidad Nacional de San Juan. \\ Av. J.I. de La Roza, 230 oeste. 5400 San Juan. República Argentina. \\ Tel.: (54-64) 222643. Fax: (54-64)228422. \\ E-mail: amacias@ffha.unsj.edu.ar; jicastro@ffha.unsj.edu.ar; cmatur@ffha.unsj.edu.ar
}

\begin{abstract}
SUMMARY
The problem about the comprehension of Physics Texts by students of the First Courses at University is the axis of analysis in this paper. Our intention is to know the learning strategies that our university students use to comprehend a scientific text. We studied the cognitive activities of the students when reading the text and the relationship between them through a global coefficient of comprehension. The results obtained let us assert that it is convenient to teach adequate strategies for the comprehension of physics texts.
\end{abstract}

\section{INTRODUCCIÓN}

La educación en los diferentes niveles y modalidades presenta problemas entre los que cabe destacar las dificultades de algunos alumnos para concretar sus aprendizajes. Atribuimos esto a numerosos factores y creemos que es conveniente señalar, entre ellos, la falta de comprensión de los textos por parte de los estudiantes, dado que éstos se utilizan muy a menudo. Los inconvenientes detectados en la comprensión de los textos, en los diferentes niveles educativos, han abierto líneas de investigación por demás interesantes (Alexander y Kulikowich, 1994; Baker, 1994; Otero, 1990; Otero, 1997; Otero y Campanario, 1990 y Sanjosé et al., 1993).

Los alumnos, con la lectura, deben abordar los conocimientos científicos y conseguir aprendizajes significativos. Esto implica que al leer deben extraer significados y construir conocimientos científicos. Para lograrlo deben hacer uso de los conocimientos previos y de proce- sos dinámicos que ayudan a la organización de la información como son: dominio semántico, sintáctico y gramatical del lenguaje, planteo de interrogantes, integración temática y logro de sus propias respuestas. Alexander y Kulikowich (1994) y De Vega (1993) señalan la complejidad cognitiva de los procesos de lectura, ya que el alumno debe operar simultáneamente en varios niveles de procesamiento, algunos conscientes y otros no conscientes (codificación de palabras, codificación sintáctica, etc.).

Así, Otero (1995) plantea los siguientes interrogantes que surgen al tratar de encontrar solución a las dificultades en la comprensión de textos científicos: «¿qué se puede hacer para que los sujetos aprendan a considerar de manera equilibrada la información científica que presenta un texto sin permitir que sus preconcepciones distorsionen el significado?, ¿cómo puede un alumno 


\section{INVESTIGACIÓN DIDÁCTICA}

aprender a elaborar espontáneamente la información cuando lee un texto científico, de tal forma que genere el mayor número de inferencias posibles e interrelacione este conocimiento con el presentado por el texto?» (p. 131). Y sugiere que esto se podría resolver con estrategias básicas de aprendizaje.

Sánchez Miguel (1993) manifiesta la importancia de instruir a los alumnos para que aprendan a comprender lo que leen. La necesidad la centra en que se detecta que hay alumnos que leen y no comprenden, otros que leen con gran fluidez y tampoco comprenden y otros que dominan el léxico e incluso la temática tratada en el texto y no retienen lo leído. Además marca que las dificultades de un sujeto de pobre comprensión pueden deberse a un problema de escasos conocimientos previos, de déficit estratégico de déficit en la autorregulación de la comprensión.

Vemos que tanto Otero como Sánchez Miguel señalan que la comprensión de los textos científicos es un proceso problemático y que se deben buscar soluciones a los inconvenientes que tienen los alumnos para que puedan abordar los textos sin dificultad. Es importante que destaquemos que el alumno debe actuar intencionalmente y poner en juego habilidades y conocimientos previos sobre el contenido del texto. Durante la lectura, debe ser capaz de realizar inferencias, representaciones mentales, elaboraciones, etc., que deben tener coherencia, evidenciando su comprensión. Estimamos que la comprensión propiamente dicha consiste en la reelaboración de los conocimientos integrados con los conocimientos previos y el recuerdo de las ideas y conceptos fundamentales. Analizando como opera el alumno podremos establecer si es un lector novato o experto.

Existen muchas variables que regulan el proceso de comprensión de textos. Éstas involucran fundamentalmente al alumno, al profesor, al contexto y al texto en sí. En base a lo expresado sobre estos últimos por autores como Alexander y Kulikowich (1994), De Vega (1993), Sánchez Miguel (1993) y Sanjosé y otros (1993), hemos elaborado un cuadro de las variables intervinientes en el proceso de comprensión de textos (Cuadro I).

Como hemos analizado, vemos que las variables que intervienen en el proceso de comprensión de textos científicos son numerosas y, aunque a veces son tratadas independientemente, se relacionan y entrecruzan, por lo que resulta su estudio muy complejo. Teniendo en cuenta todo esto, es conveniente tratar de establecer cuáles son las estrategias para la comprensión que usan nues-

Cuadro I

Variables intervinientes en el proceso de comprensión de textos.

\begin{tabular}{|l|l|}
\hline COMPONENTES & Variables que influyen y regulan el proceso de comprensión \\
\hline Profesor & $\begin{array}{l}\text { - Selección de contenidos en el libro a utilizar } \\
\text { - Elección de estrategias básicas de enseñanza } \\
\text { - Sugerencias de estrategias de aprendizaje } \\
\text { - Claridad y concisión en las instrucciones explícitas a dar a los alumnos } \\
\text { - Incentivación del interés de los alumnos para que lean textos científicos } \\
\text { - Precisión en las explicaciones que favorezcan la comprensión } \\
\text { - Etcétera }\end{array}$ \\
\hline \multirow{2}{*}{ Alumnos } & $\begin{array}{l}\text { - Conocimientos previos sobre el tema } \\
\text { - Hábitos de uso de textos } \\
\text { - Elección de estrategias de aprendizaje } \\
\text { - Interés en el tema del texto } \\
\text { - Interés en reformular sus conocimientos } \\
\text { - Etcétera }\end{array}$ \\
\hline \multirow{2}{*}{ Texto } & $\begin{array}{l}\text { - Organización (estructura) y estilo } \\
\text { - Lenguajes utilizados (lingüístico, gráfico y matemático-científico) y su corrección } \\
\text { - Inclusión de metáforas y analogías, inserción de gráficos, fotografías, comentarios aclaratorios, } \\
\text { explicaciones y elaboraciones con significación } \\
\text { - Etcétera }\end{array}$ \\
\hline Contexto & $\begin{array}{l}\text { - Condiciones implícitas: el ambiente de la clase, la predisposición de los alumnos y el profesor, etc. } \\
\text { - Condiciones explícitas: el ambiente de la clase, la predisposición de los alumnos y el profesor, etc. }\end{array}$ \\
\hline
\end{tabular}


tros alumnos de física, pues algunas variables predominarán sobre otras. Es importante equilibrar sus acciones, para lograr en éstos un rendimiento óptimo y que, al leer un texto científico, aprendan.

Pensamos que es necesario acotar el trabajo y para esto hemos circunscrito el estudio de la interacción entre las actividades cognitivas del alumno y el texto, analizando el uso que hacen los alumnos de las estrategias propuestas en una prueba.

\section{ESTUDIO DE LAS ESTRATEGIAS QUE UTILIZANLOS ALUMNOS UNIVERSITARIOS EN LA COMPRENSION DE TEXTOS DE FISICA}

Nos interesa saber qué estrategias sirven más a los alumnos en la comprensión de textos. Muchas veces una inadecuada utilización de las mismas resta efectividad a la comprensión de un texto. Creemos que podemos influir a través de la enseñanza para que ellos usen estrategias adecuadas.

Para poder analizar las estrategias que usan los alumnos y en el futuro determinar acciones, hemos diseñado una prueba en base a un texto sobre simetría y leyes de conservación extraído del libro Física (mecánica, radiación y calor) de Feynman, Leighton, y Sands (Anexo). Al mismo se le agregó un párrafo, extraído del mismo capítulo, donde se introduce el concepto de simetría. Si bien no hemos modificado el texto, cabe aclarar que ha sido sacado de dos lugares diferentes, a fin de incluir la definición del concepto de simetría, cuyo conocimiento por parte de los alumnos no podemos suponer de antemano. Cabe señalar que el texto utilizado, al igual que muchos otros que usamos a menudo en las aulas, es una traducción del inglés al castellano.

Consideramos que el texto elegido es claro en cuanto a su estructura semántica. Diversos autores (Brincones y Otero, 1994; Sánchez Miguel, 1993; van Dijk, 1980) reconocen al menos dos niveles de estructura semántica presentes en un texto que denominaremos, siguiendo a van Dijk, microestructura y macroestructura. La primera corresponde al orden de conexión, con progresión temática, de las ideas básicas del texto. La segunda se refiere al orden jerárquico, complejo y globalizador de las proposiciones. La macroestructura permite no sólo la «comprensión» del texto, sino que además organiza la información en la memoria, dando coherencia a la microestructura. La microestructura provee las ideas elementales o básicas de un texto que se relacionan por la macroestructura para dar las ideas complejas o elaboradas.

En lo que respecta a las posibles interpretaciones del texto estimamos que los conceptos que pueden presentar dificultad, por no haber tenido referencia en clases de física clásica, son los de simetría y de función de onda cuántica. Lo que suponemos como bien conocido son las leyes físicas y las leyes de conservación clásicas, dado que la muestra seleccionada está constituida por alumnos de los últimos años de los profesorados de física y de química de los cuales sólo el $43 \%$ ha cursado física moderna.

Estableceremos la comprensión que han logrado después de la lectura del texto utilizando tareas pautadas como son: resumir, contestar preguntas, interpretar gráficamente, poner título y extraer la idea principal. Lo haremos evaluando los resultados de las estrategias propuestas en la prueba, dado que son observables. Esto excluye otras tareas como, por ejemplo, realizar una comparación mental, que requieren un análisis más complejo (Wenden y Rubin, 1987).

\section{DISEÑO DE LA PRUEBA}

La prueba ha consistido en efectuar a partir de la lectura del texto las siguientes actividades:

- realizar un resumen,

- poner un título,

- extraer la idea principal,

- interpretar gráficamente lo que le sugiere el texto

- responder tres preguntas sobre el texto.

Además, a modo de autoanálisis de las estrategias de comprensión utilizadas por cada alumno, se les solicitó, de entre las opciones anteriores, señalar las que les han resultado más útiles para la comprensión.

La prueba figura en el anexo y cabe acotar que hemos permitido que mantengan el texto durante la prueba para evitar la memorización del mismo.

\section{ANÁLISIS DE LOS RESULTADOS}

A fin de evaluar a los alumnos en la comprensión del texto seleccionado, analizaremos, en primer lugar, los resultados para cada una de las actividades propuestas por separado y, luego, relacionaremos algunos de ellos buscando su estructura por análisis factorial. La prueba se aplicó a 42 estudiantes del ciclo básico universitario. Los resultados individuales de las tareas propuestas han sido calificados como buenos, regulares o malos. Los criterios que hemos adoptado para calificar los resultados en esta escala tricotómica, los señalaremos al analizar cada actividad.

\section{Resumen}

A partir del texto, los alumnos deben escoger aquellas ideas o proposiciones que consideren de relevancia y conectarlas entre ellas a fin de que su resumen sea coherente. Para evaluar este ítem procedimos a identificar en cada uno de los resúmenes elaborados por los alumnos la presencia de las ideas expuestas en el texto (proposiciones y macroestructura), su coherencia gene- 
ral en la hilación y si no hay distorsión de la información. La macroestructura se condensa en la idea de que para cada una de las reglas de simetría hay una ley de conservación correspondiente, que se pide que los alumnos reconozcan. Con respecto al análisis de la coherencia del resumen, basada en la mención del concepto de simetría, de la correlación entre reglas de simetría y leyes de conservación y algunos ejemplos del texto, se tomó el siguiente criterio para calificar el resumen: bueno, regular y malo. El alumno que ha logrado cumplimentar el resumen de esta manera se lo calificó como bueno. Si no logra todo lo exigido, regular, y malo cuando no alcanza ninguna de las condiciones exigidas.

Veamos ahora cuáles de las ideas del texto figuran en los resúmenes de los alumnos y la frecuencia con que aparecen:

- El 78\% de los alumnos explicita en el resumen la macroestructura del texto.

- Las proposiciones que más aparecen en los resúmenes elaborados por los estudiantes son las referidas a: concepto de simetría $(50 \%)$, simetría para la traslación en el espacio y conservación del momentum (43\%) y simetría para la traslación en el tiempo y conservación de la energía (28\%).

- Los más bajos porcentajes, inferiores al 15\%, corresponden a la mención de la simetría para la rotación de un ángulo fijo y corrimiento de la fase cuántica.

En el gráfico 1 vemos los resultados obtenidos.

Gráfico 1

Resultados del resumen elaborado por los alumnos.

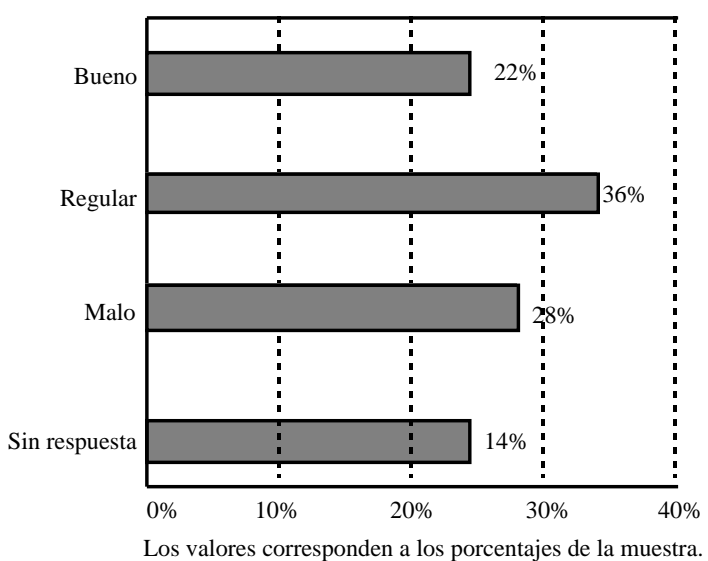

\section{Idea principal}

En este punto, los alumnos deben jerarquizar las ideas con que trabajaron en el resumen y extraer aquélla que consideren más relevante, es decir, construir la macroes- tructura del texto o al menos acercarse a ella. Para evaluar este ítem analizamos si los alumnos detectaron correctamente la jerarquía en el texto a través de la idea principal que escribieron. Destacamos que, en el original del texto seleccionado, la idea principal del mismo figura con letra cursiva. Esto ayuda al lector a extraer la misma sin mucha dificultad y constituye un recurso técnico para fijar la idea principal que, como veremos, ha sido muy efectivo. Baker (1994) expresa que la capacidad de identificar la idea principal es un aspecto crucial de la comprensión y puede ser considerada una estrategia cognitiva observable.

En el gráfico 2 se muestran los porcentajes de alumnos de la muestra agrupados según la corrección con que expresaron la idea principal del texto.

Gráfico 2

Idea principal redactada por los alumnos.

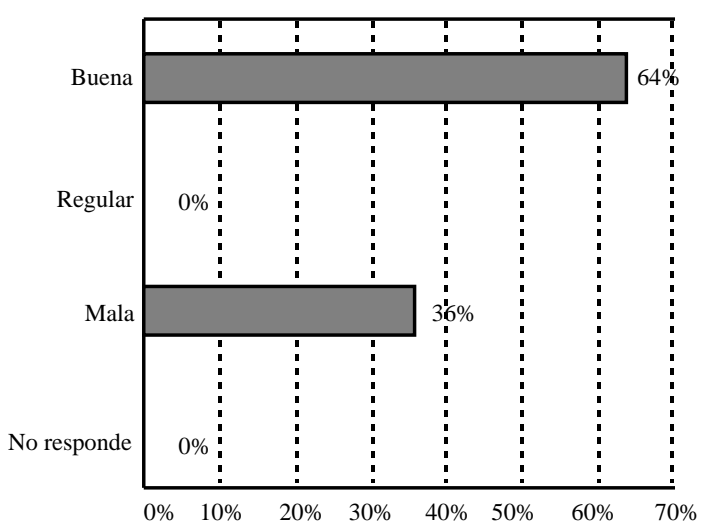

Los valores corresponden a los porcentajes de la muestra.

\section{Título}

Mayer (1986) marca la importancia de la existencia del título de un texto para lograr una mejor comprensión del mismo. Hemos considerado que, al solicitar a los alumnos esta actividad conjuntamente con la búsqueda de la idea principal, nos puede revelar el grado de comprensión logrado. Para realizar esta actividad, el alumno debe interrelacionar globalmente las ideas del texto y determinar de qué trata el mismo.

El análisis de las pruebas en este caso se aboca a determinar la coherencia entre el título colocado y lo expresado globalmente por el texto. Evaluamos como bueno un título que reúne las condiciones señaladas; regular, cuando no cumple estrictamente las condiciones de un buen título; y malo, cuando es incoherente. Los resultados obtenidos se muestran en el gráfico 3. 
Gráfico 3

Coherencia del título puesto por los alumnos.

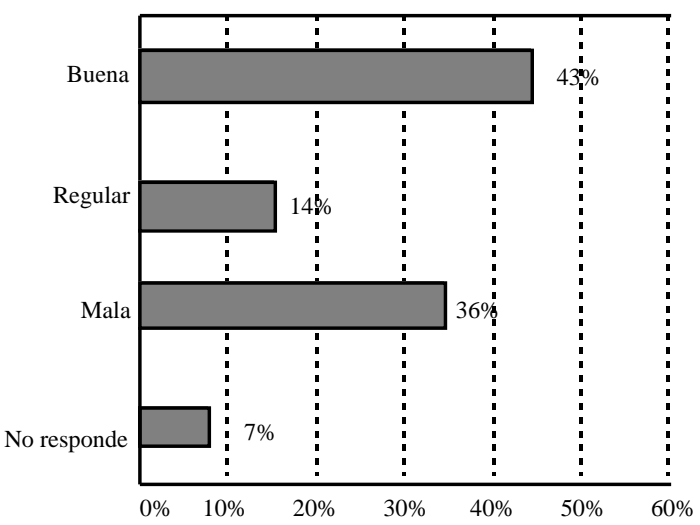

Los valores corresponden a los porcentajes de la muestra.

\section{Interpretación gráfica}

Hemos considerado importante que los estudiantes realicen una interpretación gráfica del texto. Algunos alumnos confeccionaron dibujos y otros esquemas conceptuales. En el caso de los dibujos evaluamos la relación con el texto desde dos aspectos: concepto de simetría y operaciones de simetría. La interpretación gráfica debía contener indicación no sólo de la simetría sino también de la operación de invariancia para ser evaluado como bueno.

En los esquemas conceptuales tuvimos en cuenta la coherencia interna. En ambos casos valoramos según tres niveles (bueno, regular o malo), tal como procedimos anteriormente. Los resultados se muestran en el gráfico 4.

Gráfico 4

Interpretación gráfica realizada por los alumnos.

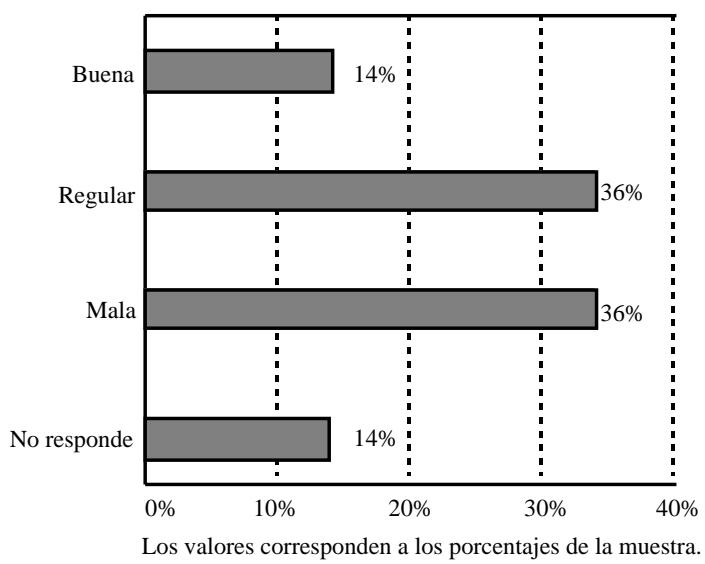

ENSEÑANZA DE LAS CIENCIAS, 1999, 17 (3)

\section{Cuestionario}

El uso de los cuestionarios, tanto en un proceso de estudio de un tema como de evaluación del mismo, formulados en función de un texto puede convertirse en una eficaz herramienta de aprendizaje y de evaluación. Para verificar la comprensión que los alumnos hicieron del texto y comparar luego con los resultados anteriores, les formulamos tres preguntas referidas a: concepto de simetría, reglas u operaciones de simetría y conocimiento de otro tipo de simetrías (no mencionadas en el texto).

El análisis de los resultados lo hicimos siguiendo el criterio utilizado anteriormente (bien, regular y mal para cada pregunta). Los mismos se pueden visualizar en el gráfico 5 .

Gráfico 5

Respuestas a las preguntas referidas al texto.

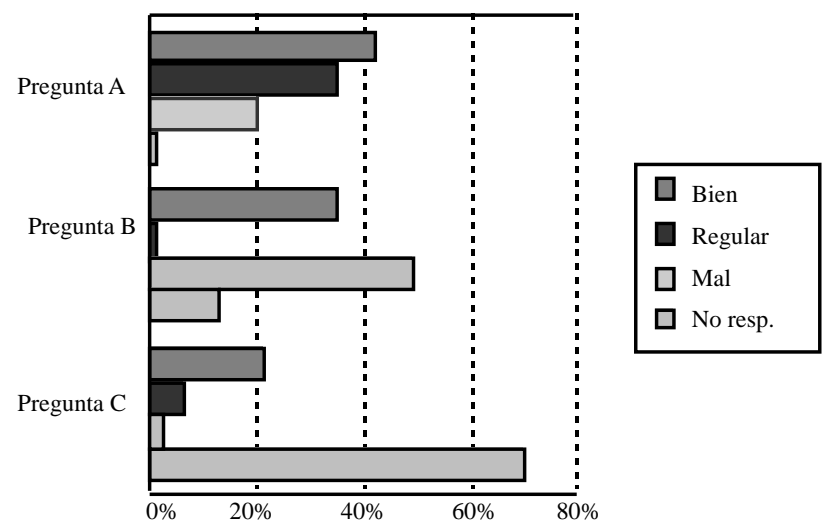

Los valores están expresados en porcentajes de la muestra.

\section{Vinculación de los resultados. Coeficiente global de comprensión}

Analizados estadísticamente los datos de la prueba, se han obtenido los coeficientes de correlación para el rendimiento y coherencia de los alumnos en la realización de cada tarea propuesta: confección del resumen (R), extracción de la idea principal (I), adjudicación de título (T), interpretación gráfica de lo sugerido por el texto $(\mathrm{G})$ y respuesta a las preguntas del cuestionario $(\mathrm{P})$. Cada estudiante ha sido calificado (bueno, regular o malo con valuación 2,1 y 0 ) en cada ítem (R, I, T, G y P), siendo posible correlacionar cada par (RI, RT, etc.) por medio de las calificaciones de todos los estudiantes. Por ejemplo, el coeficiente de correlación de Pearson entre R e I para la muestra seleccionada es $r_{R I}=0,372$.

Así, es posible establecer la siguiente matriz de correlaciones: 


\begin{tabular}{|c|c|c|c|c|c|}
\hline & $\mathbf{R}$ & I & $\mathbf{T}$ & $\mathbf{G}$ & $\mathbf{P}$ \\
\hline $\mathrm{R}$ & 1 & 0,372 & $-0,300$ & 0,506 & 0,266 \\
\hline I & 0,372 & 1 & 0,322 & 0,460 & 0,498 \\
\hline $\mathrm{T}$ & $-0,300$ & 0,322 & 1 & $-0,107$ & 0,210 \\
\hline G & 0,506 & 0,460 & $-0,107$ & 1 & 0,063 \\
\hline $\mathrm{P}$ & 0,266 & 0,4988 & 0,210 & 0,063 & 1 \\
\hline
\end{tabular}

Considerando correlaciones altas $(1 \geq \mathrm{r} \geq 0,67)$, medias $(0,67 \geq r \geq 0,33)$ y bajas $(0,33 \geq r \geq 0)$, podemos observar que sólo hay correlaciones medias.

Toda vez que existe una matriz de correlaciones, es posible reducir el número de variables (cinco en nuestro caso) buscando combinaciones lineales (llamadas factores) independientes entre sí y con significación que se determinó posteriormente a la realización del cálculo.

Hemos realizado el análisis factorial con el criterio de rotar los ejes factoriales hasta hacer coincidir uno de ellos con la variable $\mathrm{T}$ por ser la de menores correlaciones. Los resultados nos muestran que las correlaciones son explicadas por cuatro factores (F1, F2, f y fr); la matriz factorial correspondiente es:

\begin{tabular}{|c|c|c|c|c|}
\hline & F1 & F2 & $\mathbf{f}$ & fr \\
\hline $\mathrm{R}$ & $-0,0037$ & 0,7411 & $-0,3691$ & 0,1506 \\
\hline I & 0,0040 & 0,7324 & 0,3967 & $-0,1618$ \\
\hline $\mathrm{T}$ & 0,0000 & 0,0000 & 0,8117 & 0,0000 \\
\hline $\mathrm{G}$ & $-0,4294$ & 0,6563 & $-0,1328$ & $-0,2082$ \\
\hline $\mathrm{P}$ & 0,6101 & 0,5129 & 0,2583 & $-0,1079$ \\
\hline
\end{tabular}

Las comunalidades, es decir, la parte de la varianza de cada variable explicada por los factores, que se calculan como la suma de los cuadrados de los elementos de cada fila de la matriz factorial y los porcentajes de las mismas explicados por los tres primeros factores, son:

Comunalidades

\% cubierto por F1, F2 y f

\begin{tabular}{|l|lc|}
\hline $\mathrm{R}$ & 0,708 & $97 \%$ \\
\hline $\mathrm{I}$ & 0,720 & $96 \%$ \\
\hline $\mathrm{T}$ & 0,659 & $100 \%$ \\
\hline $\mathrm{G}$ & 0,676 & $94 \%$ \\
\hline $\mathrm{P}$ & 0,714 & $98 \%$ \\
\hline
\end{tabular}

Inferimos de la última columna de la tabla anterior que al factor fr (factor residual) podemos no considerarlo en el análisis.
La figura 1 muestra los ejes factoriales y la composición factorial de cada variable.

Figura 1

Componentes factoriales F1 (factor de autorregulación), F2 (factor conceptual), -f (factor de capacidad de síntesis) de las variables R, I, T, G y P.

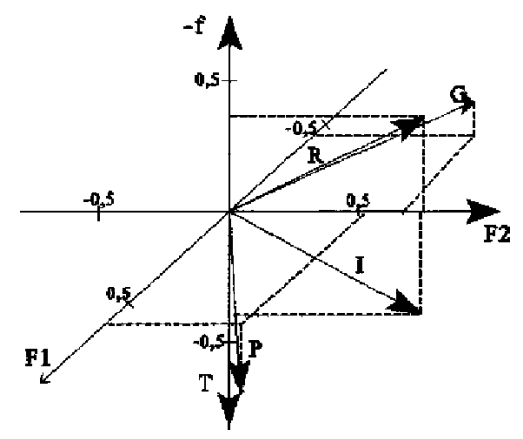

$$
\begin{array}{lrrrr}
\mathrm{R}= & & 0,74 \mathrm{~F} 2 & -0,37 \mathrm{f} & (+0,15 \mathrm{fr}) \\
\mathrm{I}= & & 0,73 \mathrm{~F} 2+0,40 \mathrm{f} & (-0,16 \mathrm{fr}) \\
\mathrm{T}= & & +0,81 \mathrm{f} & \\
\mathrm{G}= & -0,43 \mathrm{~F} 1+0,66 \mathrm{~F} 2 & -0,13 \mathrm{f} & (-0,21 \mathrm{fr}) \\
\mathrm{P}= & 0,61 \mathrm{~F} 1+0,51 \mathrm{~F} 2 & +0,26 \mathrm{f} & (0,11 \mathrm{fr})
\end{array}
$$

Por otra parte, con el criterio $\mathrm{r} \geq 0,37$, basándonos en los datos anteriores y en la matriz de correlaciones, se puede establecer el grafo que se muestra en la figura 2, donde indicamos los tres factores de los que depende la comprensión.

En este gráfico se observa la vinculación por correlaciones fuertes entre R, G e I; por su parte, $P$ sólo está vinculado con I con una correlación intermedia, mientras que $\mathrm{T}$ permanece aislado, sin correlaciones notables. Los valores de la variable T son explicados mediante el único factor $\mathrm{f}$; $\mathrm{R}$ e I son explicadas por $\mathrm{f}$ y $\mathrm{F} 2$, mientras que $\mathrm{G}$ y $\mathrm{P}$ son explicadas por f, F2 y F1.

$\mathrm{Si}$ analizamos individualmente los factores, vemos que se pueden interpretar (posteriormente a la realización de los cálculos) según las siguientes consideraciones:

a) El factor -f aumenta en la medida en que la tarea de los alumnos exige una mayor capacidad de síntesis; la ortogonalidad de $\mathrm{T}$ con respecto a las otras variables indica que existe una incapacidad para inducir un concepto globalizador del texto leído. Llamaremos a -f factor de capacidad de síntesis, (el cambio de signo se impone para evitar hablar de una «incapacidad» de síntesis, verdadero significado de f). 
Figura 2

Grafo de correlación entre variables y factores que explican cada variable.

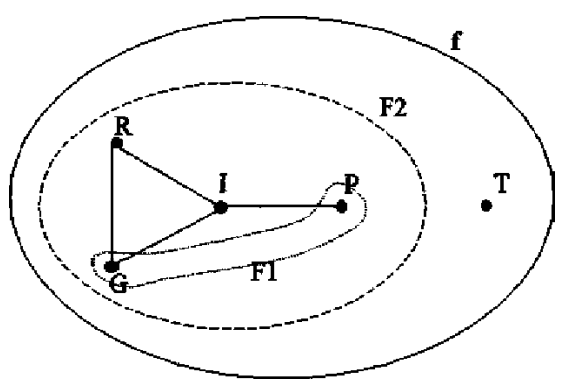

b) El factor F1 lo relacionamos con la iniciativa propia del estudiante-lector para realizar una tarea de aplicación de los conceptos adquiridos; en tal sentido, la tarea más pautada es la de responder preguntas específicas $(\mathrm{P})$ y la más libre es la de interpretar gráficamente el texto (G); al factor F1 lo denominaremos factor de autorregulación.

c) El factor F2 consideramos que se relaciona con las adquisiciones conceptuales realizadas por el alumno a partir del texto. El factor F2 lo denominaremos factor conceptual.

Podemos inferir que la ausencia de $\mathrm{F} 2$ en la variable $\mathrm{T}$ indica que los alumnos no se guían por los conceptos contenidos en el texto para adjudicarle un título, aún cuando la coherencia del título puesto por los alumnos (ver gráfico correspondiente) tiene un rendimiento global (no individual) que es del $43 \%$. Esto no indica comprensión, puesto que las respuestas individuales no están correlacionadas entre sí con las otras tareas (ver la matriz de correlación). Así mismo, observamos que este factor es el predominante en las tareas restantes.

Concluimos de todo el análisis anterior que en el proceso de comprensión llevado a cabo por nuestros alumnos intervendrían tres factores principales. El factor conceptual, preponderante, que está condicionado con la estructura del texto y su capacidad para transmitir al lector nuevos conocimientos potencialmente significativos. A éste se suma un factor de autorregulación, dependiente del alumno, que muestra que el lector modifica lo expresado en el texto, añadiendo una cuota de originalidad e imaginación a lo leído (Schuder et al., 1995). Por último, existe un factor funcional de capacidad de síntesis que influye en el grado de comprensión de lo leído, lo cual implica previsibles dificultades en el recuerdo a largo plazo (Slater y Graves, 1995).

Hemos establecido además un coeficiente global de comprensión (C) como el promedio valorado de los rendimientos de los alumnos en las diversas tareas. Obtuvimos $C=0,45$ con un desvío standard de 0,25 . Este coeficiente así definido es análogo, si bien es distinto, a la Comprehension Monitoring Ability (CMA) usada por Otero, Campanario y Hopkins (1992). La CMA se refiere a procesos metacognitivos (relacionados con el conocimiento que un sujeto tiene de su propia comprensión) mientras que $\mathrm{C}$ lo hace al aprendizaje cognitivo del texto. De hecho, el valor de C (cuyos valores de referencia son 1 para el lector experto y 0 para un lector que no comprende) coincide con los valores obtenidos para la CMA reducidos a escala $0-1$ de alumnos españoles de grado $12^{\circ}$ (aproximadamente 18 años), lo cual sugiere una posible relación entre la CMA y C.

Hemos detectado que la existencia de una estructura conceptual significativa previa influye en una mejor comprensión del texto. Los alumnos que han cursado física moderna superan en su comprensión promedio a los que no lo hicieron (el coeficiente $C$ vale 0,57 en un caso y 0,37 en el otro caso). De aquí se desprende la conveniencia de reconocer de modo explícito las estructuras conceptuales previas del lector y también del autor del texto, tanto por parte del alumno como por parte del profesor en su accionar.

\section{Opiniones}

En este punto solicitamos a los alumnos que identificaran aquellas actividades que más les ayudaron a comprender el texto.

Los resultados obtenidos se muestra en la siguiente tabla.

\begin{tabular}{|l|c|}
\hline Actividad & $\begin{array}{c}\text { Porcentaje de alumnos } \\
\text { de la muestra } \\
\text { que mencionan } \\
\text { dicha actividad }\end{array}$ \\
\hline Elaboración de resumen & $71 \%$ \\
\hline Extracción de la idea principal & $86 \%$ \\
\hline Colocación de título & $7 \%$ \\
\hline Interpretación gráfica & $21 \%$ \\
\hline Respuesta al cuestionario & $21 \%$ \\
\hline
\end{tabular}

Observamos una coincidencia entre el análisis realizado anteriormente (y mostrado en el grafo) y las opiniones de los alumnos.

\section{CONCLUSIONES}

El análisis de las pruebas aplicadas para el estudio de las estrategias de aprendizaje de los alumnos nos permite arribar a algunas conclusiones interesantes. 
Las estrategias de aprendizaje utilizadas por los alumnos han sido acotadas, en parte, por las actividades propuestas en la prueba. Es por esto que las conclusiones que sacamos se refieren a ellas aún cuando los alumnos podrían haber utilizado otras estrategias que no investigamos en esta ocasión. Al pautar, detectamos que hay estrategias más efectivas en cuanto a interpretación y análisis de texto. Así, por ejemplo, en función de los resultados podemos establecer que extraer la idea principal es más eficaz que poner un título.

Es evidente a partir de los resultados citados anteriormente que, en general, hay una pobre comprensión del texto en los alumnos, que hace necesario que investigadores y profesores debamos mejorar las estrategias usadas hasta el momento, que no resultan totalmente eficaces en la educación sistemática.

Sabemos que la comprensión de un texto es una actividad guiada y controlada por el propio lector, quien debe interpretar y organizar la información en su mente, a partir de sus conocimientos previos y del conocimiento surgido del texto. En el caso de los alumnos, protagonistas del proceso de aprendizaje, depende de ellos, en definitiva, la construcción de sus conocimientos. Sin embargo, esta construcción no se manifiesta, salvo en muy pocos casos, como una determinación propia. Para esto se requiere la intervención del docente que con su accionar promueve el acercamiento del alumno al libro y además debería enseñar estrategias de aprendizaje,

\section{REFERENCIAS BIBLIOGRÁFICAS}

ALEXANDER, P.A. y KULIKOWICH, J.M. (1994). Learning from Physics Text: A Sinthesis of Recent Research. Journal of Research in Science Teaching, 31(9), pp. 895-911.

BAKER, L. (1994). Metacognición, lectura y educación científica, en Minnick Santa, C. y Alvermann, D.E. (comp.). Una didáctica de las ciencias, procesos y aplicaciones. Buenos Aires: Aique.

BRINCONES, I. y OTERO, J. (1994). Students' Conceptions of the Top-Level Structure of Physics Texts. Sciencie Education 78(2), pp. 171-183.

FEYNMAN, R.P., LEIGHTON, R.B. y SANDS M. (1987). Física. Volumen 1 (Mecánica, radiación y calor). EEUU: Addison-Wesley Iberoamericana. como, por ejemplo: realizar actividades de prelectura, identificar microestructura y macroestructura en el texto, resumir, extraer la idea principal, etc.

El trabajo de Otero, Campanario y Hopkins (1992), sobre el CMA y el presente trabajo, donde definimos el coeficiente global de comprensión, muestra analogías suficientes como para alentar la expectativa de encontrar una relación entre ambos coeficientes y de profundizar el estudio de los factores intervinientes en el proceso de comprensión cuando los lectores son expertos. En efecto, debemos tratar de que el alumno novato, que ya cuenta con un estado inicial (conocimientos, habilidades y estrategias propias) se convierta en competente en la comprensión; para ello, deben establecerse los medios para el paso de una situación a otra, y esto se logra con estrategias que actúen sobre los factores característicos del novato y los transformen en los del experto. Este trabajo (determinación de los factores que intervienen en la comprensión de un experto) está actualmente en etapa de realización.

\section{AGRADECIMIENTOS}

El presente trabajo ha sido realizado con el apoyo de la Universidad Nacional de San Juan, que avala y subsidia las investigaciones realizadas. Así mismo agradecemos el asesoramiento y las sugerencias del Dr. José Otero de la Universidad de Alcalá de Henares.
MAYER, R.E. (1986). Pensamiento, resolución de problemas y cognición. Barcelona: Paidós.

OTERO, J. (1990). Variables cognitivas y metacognitivas en la comprensión de textos científicos: el papel de los esquemas y el control de la propia comprensión. Enseñanza de las Ciencias, 8(1), pp 17-22.

OTERO, J. y CAMPANARIO J.M. (1990). Comprehension evaluation and regulation in learning from science texts. Journal of Research in Science Teaching, 27(5), pp. 447-460.

OTERO, J., CAMPANARIO, J.M. y HOPKINS, K.D. (1992). The relationship between academic achievement and metacognitive comprehension monitoring ability of Spanish 
secondary school students. Educacional and Psychological Measurement, 52, pp. 419-430.

OTERO, J. (1995). Estrategias básicas de aprendizaje frente a contenidos y métodos en la enseñanza de la física. Tarbiya, 10 , pp. 127-133.

OTERO, J. (1997). El conocimiento de la falta de conocimiento de un texto científico. Alambique, 11, pp. 15-22.

SÁNCHEZ MIGUEL, E. (1993). Los textos expositivos. Estrategias para mejorar su comprensión. Madrid: Santillana.

SANJOSÉ LÓPEZ, V., SOLAZ PORTALÉS, J.J. y VIDALABARCA GÓMEZ, E. (1993). Mejorando la efectividad instruccional del texto educativo en ciencias. Enseñanza de las Ciencias, 11(2), pp. 137-148.
SLATER, W.H. y GRAVES, M.F. (1995). Investigaciones sobre el texto expositivo: aportes para los docentes, en Muth, K.D. (comp.). El texto expositivo. Estrategias para su comprensión. Buenos Aires: Aique Grupo editor, SA.

SCHUDER, T., CLEWELL S.F. y JACKSON, N. (1995). Captar lo esencial en un texto expositivo, en Muth K.D. (comp.). El texto expositivo. Estrategias para su comprensión. Buenos Aires: Aique.

VAN DYCK, T.A. (1980). Texto y contexto. Semántica y pragmática del discurso. Madrid: Ediciones Cátedra, SA.

VEGA DE, M. (1993). Introducción a la Psicología Cognitiva. Madrid: Alianza.

WENDEN, A. y RUBIN, J. (1987). Learner Strategies in language learning. Cambridge: Prentice-Hall.

[Artículo recibido en octubre de 1997 y aceptado en junio de 1998.] 


\title{
ANEXO
}

\author{
NOMBRE Y APELLIDO: \\ CARRERA: \\ CURSO: \\ ¿Ha cursado física moderna?: \\ EDAD:
}

\section{Instrucciones generales}

- Lee las consignas con detenimiento.

- Trabaja con prolijidad.

- Escribe todas tus respuestas en estas hojas.

Ante todo, ¿qué es simetría? ¿Cómo puede una ley física ser «simétrica»? El problema de definir simetría es interesante y ya hemos apuntado que Weyl dio una buena definición, cuya esencia es: una cosa es simétrica si hay algo que podamos hacer con ella de tal modo que después que lo hemos hecho parece la misma cosa que antes. Por ejemplo, un jarrón simétrico es tal que reflejándolo o girándolo tendrá el mismo aspecto que antes...

Las simetrías de las leyes físicas son muy interesantes a este nivel, pero resulta, al fin, que son aún más interesantes y excitantes cuando llegamos a la mecánica cuántica. Por una razón que no podemos aclarar al nivel de la presente discusión - un hecho que la mayoría de los físicos aún encuentran algo desconcertante, una cosa muy profunda y bella-, es que, en mecánica cuántica, para cada una de las reglas de simetría hay una ley de conservación correspondiente: hay una conexión definida entre las leyes de conservación y las simetrías de las leyes físicas. Sólo podemos decir esto por ahora sin ningún intento de explicación.

Por ejemplo, el hecho de que las leyes son simétricas para la traslación en el espacio, cuando añadimos los principios de mecánica cuántica, resulta que significa que el momentum se conserva.

Que las leyes son simétricas frente a una traslación en el tiempo significa en mecánica cuántica que la energía se conserva.

La invariancia frente a una rotación de un ángulo fijo en el espacio corresponde a la conservación del momentum angular. Estas conexiones son cosas muy interesantes y bellas, entre las cosas más bellas y profundas de la física.

Entre paréntesis, hay una cantidad de simetrías que aparecen en mecánica cuántica que no tienen análogo clásico, que no tienen método de descripción en física clásica. Una de ellas es la siguiente: Si $\psi$ es la amplitud de algún proceso, sabemos que el cuadrado del módulo de $\psi$ es la probabilidad de que el proceso ocurra. Ahora bien, si algún otro tiene que hacer sus cálculos, no con esta $\psi$, sino con una $\psi^{\prime}$ que difiera simplemente en un cambio de fase (digamos que $\Delta$ sea una constante y multipliquemos $\mathrm{e}^{\mathrm{i} \Delta}$ por la vieja $\psi$ ), el cuadrado del módulo de $\psi^{\prime}$, que es la probabilidad del evento, es, entonces, igual al cuadrado del módulo de $\psi$ :

$$
\psi^{\prime}=\psi \mathrm{e}^{\mathrm{i} \Delta} ;\left|\psi^{\prime}\right|^{2}=|2|^{2}
$$

Por lo tanto, las leyes físicas no varían si la fase de la función de onda está corrida en una constante arbitraria. Esta es otra simetría. Las leyes físicas deben ser de tal naturaleza que un corrimiento de la fase cuántica no altere nada. Como acabamos de decir, en mecánica cuántica hay una ley de conservación para cada simetría. La ley de conservación que se relaciona con la fase cuántica parece ser la conservación de la carga eléctrica. ¡Todo esto en conjunto es un negocio muy interesante!

\section{Consignas}

1. Lee atentamente el texto anterior las veces que creas conveniente hasta que lo comprendas.

2. Elabora un resumen del texto.

3. ¿Cuál es, según tu criterio, la idea principal del texto?

4. ¿Qué título le pondrías al texto?

5. Expresa gráficamente lo que te sugiera el texto.

6. a) ¿Qué entiendes por simetría? b) ¿Cuáles son las transformaciones de simetría que tienen análogo clásico?, ¿y las que sólo se presentan en mecánica cuántica? c) ¿Podrías nombrar otras simetrías que no estén mencionadas en el texto?

7. ¿Cuáles de las actividades realizadas te ayudaron más a comprender el texto? (Indica el núm. de la consigna correspondiente.) 